\title{
Mild COVID-19 manifestation in multiple risk factor patient on methotrexate, who had been treated with UVB phototherapy and had sufficient plasma 25-OH-vitamin D3 level
}

\author{
Martina Kralj ${ }^{1}$ and Hrvoje Jakovac ${ }^{2}$ \\ ${ }^{1}$ General Hospital Karlovac \\ ${ }^{2}$ University of Rijeka Faculty of Medicine
}

January 31, 2021

\begin{abstract}
We report mild COVID-19 manifestation in high-risk patient with sufficient plasma 25-OH-Vitamin D3 level. Given the global pandemic of vitamin D deficiency, as well as its likely beneficial effects during SARS-CoV-2 infection, report highlights importance of routine 25-OH-Vitamin D3 measurement, either for clinical course prediction or deciding on supplementation.
\end{abstract}

\section{Introduction}

During the recent COVID-19 pandemic, several well defined conditions have been recognized as a risk factors worsening disease course and outcome. ${ }^{1,2}$ The most important risk factors which can lead to severe COVID-19 symptomatology are obesity, diabetes mellitus, hypertension, age over 65 years and immunosuppressive therapy. ${ }^{3}{ }^{4}$ These conditions are thought to affect host responses to infection by various mechanisms, enhancing and accelerating the harmful pathophysiological processes. ${ }^{5}$ The COVID-19 pathogenesis upon SARS-CoV-2 infection is characterized by initial viral evasion from targeted immune response, followed by excessive and undirected immune activation causing subsequent hyper inflammatory response ${ }^{6}$. Patients with risk factors more likely react with extremely excessive systemic inflammation resulting with cytokine storm which is considered as an executive pathophysiological mechanism responsible for severe disease manifestations, such as acute respiratory distress syndrome (ARDS), diffuse endothelial damage with accelerated thrombogenesis and multiple organ dysfunction syndrome (MODS) ${ }^{6}$. In most groups of risk patients, common mechanism favoring development of excessive inflammation and cytokine storm is persistently present proinflammatory milieu ${ }^{7}$. Such chronic proinflammatory state can arises either due to hypersecretion of proinflammatory adipokines in obese patients ${ }^{7}$, lack of anti-inflammatory signaling in diabetic and insulinresistant patients ${ }^{7,8}$ or dysregulation of renin angiotensin system (RAS) with increased proinflammatory angiotensin II (Ang II) production in hypertensive patients ${ }^{6,9,10}$. Patients on immunosuppressive therapy are considered to be at risk due to the facilitated viral multiplication and dissemination during the initial phase, enabling in turn more vigorous ignition of hyperinflammatory phase ${ }^{6,10,11}$. In the other hand, hyperactive inflammatory processes could be, in some extent, kept under control by immunosuppressants ${ }^{12}$, but conclusions and recommendations on their use in COVID-19 patients are inconsistent and unclear. Moreover, most intriguing obscurity during COVID-19 pandemic - enormous variability of clinical presentation and unpredictability of outcome, either in previously healthy or in at-risk patients, still remains enigmatic.

In effort to prevent adverse outcomes of SARS-CoV-2 infection, growing attention is paid to potentially protective factors alleviating disease severity, among which vitamin D3 is currently being in the spotlight. Namely, many "pre-corona" studies have found antiviral, immunomodulatory, and anti-inflammatory properties of vitamin D3 ${ }^{13}$, suggesting this data could be extrapolated to SARS-CoV-2 infection ${ }^{14,15}$. Assumptions 
on beneficial effects of vitamin D3 in COVID-19 patients have recently been verified by several convincing clinical and interventional studies ${ }^{16-24}$. The importance of this topic is highlighted by data showing that the global pandemic of hypovitaminosis Dis being ongoing concomitantly with the COVID-19 pandemic ${ }^{25-28}$.

Here, we report mild clinical COVID-19 presentation in high-risk patient with the satisfactory vitamin D3 level upon UVB phototherapy treatment, pointing to the vitamin D3 as a likely positive modulator of disease course, as well as to potential value of routine 25-OH-Vitamin D3 measurement in COVID-19 patients.

\section{Case report}

66-years old Caucasian male sought medical advice due to slightly elevated body temperature (up to 37.50C), nausea without vomiting and occasional, mild, and dry cough during the last 2 days. He did not complain of dyspnea, shortness of breath, myalgias or arthralgias during that period. He did not notice any loss of sense of smell or taste. He denied recent traveling or contacts with symptomatic persons. On admission patient was alert, conscious, oriented, and independently movable and he was seeming to be in good general condition. Physical examination revealed pulse rate of $100 \mathrm{bpm}$, respiratory rate of $16 \mathrm{bpm}$, arterial blood pressure of $147 / 75 \mathrm{~mm} \mathrm{Hg}$ and axillary measured body temperature of 37.50C. Chest auscultation disclosed clear breath sounds without obvious moist/dry rales or crackles, as well as normal heart sounds without audible murmurs. Abdomen was soft and painless on palpation, and no discomfort was elicited. Neither organomegaly nor palpable pathological masses were detected. The patient was obese, with a body weight of $99.5 \mathrm{~kg}$ and body height of $167 \mathrm{~cm}$, resulting in a body mass index (BMI) of 35.7. He had been passionate smoker for a long period of time, and he stopped smoking 6 years ago. Before 25 years, patient has been diagnosed with psoriasis. Severe psoriatic arthritis has occurred 6 years ago, and since that time he has been continuously receiving methotrexate at the dose of $15 \mathrm{mg} /$ week subcutaneously. Folic acid in the dose of 5 mg twice weekly was prescribed to prevent folate deficiency. Three months before the actual presentation cutaneous psoriatic symptomatology exacerbated, and the patient was undergoing to UVB narrow band phototherapy lasting for two months.

Patient was diagnosed with arterial hypertension 8 years ago, and diagnosis of type 2 diabetes mellitus (T2DM) has been established 6 years ago. To treat these conditions, he has been regularly taking losartan with hydrochlorothiazide $(50 \mathrm{mg}+12.5 \mathrm{mg}$ once a day) and metformin (500 $\mathrm{mg}$ three times a day).

The patient was referred to COVID-19 testing, and nasopharyngeal swab was taken. Detection of viral RNA by GeneXpert SARS-CoV-2 RT-PCR assay (Cepheid) resulted in a positive finding. A chest X-ray was done and finding was unremarkable, without any opacity indicating infiltration or consolidation. Blood oxygen saturation ( $\mathrm{SpO} 2$ ) measured by pulse oximeter was $95 \%$. Subsequent routine laboratory tests found slightly elevated C-reactive protein $(7 \mathrm{mg} / \mathrm{L})$; increased erythrocyte sedimentation rate $(50 \mathrm{~mm} / \mathrm{hr})$; normal white blood cell count $\left(4.8 \times 10^{9} / \mathrm{L}\right)$ with lymphopenia $\left(1.02 \times 10^{9} / \mathrm{L}\right)$; decreased red blood cell count $\left(4.06 \times 10^{12} / \mathrm{L}\right)$, hematocrit $(0.387)$ and hemoglobin concentration $(132 \mathrm{~g} / \mathrm{L})$; hyperglycemia $(10.1 \mathrm{mmol} / \mathrm{L})$, hypercreatininemia $(134 \mu \mathrm{L} / \mathrm{L})$ and hypertriglyceridemia $(1.8 \mathrm{mmol} / \mathrm{L})$. Semi quantitative dipstick urinalysis indicated mild proteinuria $(1+)$. Further laboratory investigations revealed normal plasma 25-OH-vitamin D3 concentration of $92.2 \mathrm{nmol} / \mathrm{L}$ (normal range $50.0-125.0 \mathrm{nmol} / \mathrm{L}$ ). All laboratory parameters, alongside with corresponding normal ranges, are shown in the Table 1.

Considering the mild clinical picture, no therapy was introduced, and all previous medications were continued. Given the occupancy of hospital facilities with severe cases, the patient was monitored on an outpatient basis. His condition did not worse and presenting symptoms completely subsided within 3 days. Twenty days after the initial presentation SARS-CoV-2 RT-PCR test was negative, and serological enzyme-linked fluorescence assay (ELFA) shoved presence of SARS-CoV-2 IgG (33.16) and IgM (5.09) in the patient's serum. During the next month, the patient felt well, not complaining of fatigue or exhaustion, and dermatological, rheumatological and internistic symptoms remained stationary.

\section{Discussion}

Almost immediately after the pandemic outbreak in China, greater vulnerability and higher incidence of 
severe clinical presentation and fatal outcome were observed in patients with particular preexisting conditions, especially in persons aged $>65^{1,2}$. Numerous studies have shown that the greatest risk of severe COVID19 course/manifestation is posed by the presence of either diabetes mellitus, obesity, arterial hypertension or immunosuppression / immunodeficiency ${ }^{3-8}$. Severe COVID-19 manifestations include development of extensive bilateral pneumonia, ARDS and endothelial damage accompanied with accelerated thrombogenesis, which can all lead to the respiratory and multiorgan failure, being major clinical concern due to intensive care units overcrowding and high mortality rate ${ }^{4,6}$.

According to the current knowledge, the basic pathogenetic mechanisms responsible for the propensity to severe COVID-19 course in the patients at risk could be reduced to a common denominator relating to facilitation and reinforcement of inflammatory processes. Inappropriately excessive and uncontrolled inflammation more likely can result with the development of autodestrucitive cytokine storm, which is considered to be the final effector mechanism of tissue damage ${ }^{4,6,10,11}$. Obesity, insulin resistance and diabetes mellitus, as well as hypertension, are characterized by the presence of persistent low-intensity inflammation, which may be decisive underlying factor amplifying and perpetuating viral-induced inflammatory response ${ }^{4-11}$. Some authors also suggest pathogenetic involvement of immunogenic damage-associated molecular patterns (DAMPs), released as a consequence of preexisting disease ${ }^{29}$. Immunosuppression is thought to favor more severe disease development by permitting faster viral replication resulting in extensive direct viral-mediated tissue damage ${ }^{4,6,11}$. In the other hand, immunosuppression can mitigate excessive inflammation. There are studies showing no increased risk of severe COVID-19 manifestation in patients on immunosuppressive therapy, including methotrexate ${ }^{30,31}$, as well as reports that emphasize severe COVID-19 course in such patients ${ }^{32,33}$. Accordingly, available data on the impact of variety of immunosuppressant therapy regarding COVID-19 clinical course are inconclusive yet ${ }^{30,34}$. Both, insufficient directed immune response as well as unpurposeful inflammatory overactivity are assumed to increase possibility of severe COVID-19 manifestations in the elderly patients, as aging is known to impair considerably the efficacy of immunoregulatory mechanisms ${ }^{35,36}$. The probability of severe COVID-19 increases with age and number of preexisting pathological conditions and is particularly high in patients with older age and more risk comorbidities ${ }^{37}$, indicating a cumulative effect of the risk factors.

Here we described a mild COVID-19 course, without any concerning symptoms or clinical signs, and with a favorable outcome in the patient with multiple conditions considered as risk factors: obesity, T2DM, hypertension, age $>65$ and immunosuppressive therapy. Laboratory findings of the proteinuria and hypercreatininemia also suggest renal function impairment, probably due to diabetic nephropathy development. Chronic renal failure was also found to be independently associated with poor clinical outcome ${ }^{38}$. Besides, given the long-standing history of psoriasis in our patient (25 years), we also point out that several studies have found increased risk of serious infection (especially cutaneous and respiratory) in psoriatic patients independently on the therapy and other comorbidities ${ }^{30,39,40}$. The risk of serious infection in psoriatic patients was found to increase further with the severity of the disease ${ }^{39}$ and the presence of either diabetes mellitus, obesity, age $>60$ years ${ }^{41,42}$ or history of smoking ${ }^{42}$. Taking into account the recent cutaneous exacerbation (three months before the actual presentation; treated by UVB phototherapy), our patient had all of the above conditions that may additionally increase the risk of severe infection in the context of psoriasis per se

Laboratory findings in our patients, showing normal leukocyte count and only slightly elevated inflammatory markers, are in line with a mild clinical picture. The exception is lymphopenia, which has been found to be associated with severe COVID-19 cases $^{37,38}$, but in presented patient lymphopenia very likely may be a consequence of long-term treatment with methotrexate, which is known to inhibit DNA synthesis and cause bone marrow toxicity ${ }^{43,44}$. Such causality can also be attributed to the anemia, found in our patient. Although there is a paucity of data on clear relationship between anemia and COVID-19 severity, one metaanalysis found an association of anemia with severe manifestation of COVID19, but not with mortality rate $^{45}$.

We would like to emphasize the sufficient plasma 25-OH-vitamin D3 (calcifediol, precursor of an active form 
of the vitamin D3) level in the presented patient, which could have had, at least in part, positive impact on the disease course and outcome. Namely, since time when the possible favorable effects of vitamin D3 in COVID-19 patients have been proposed ${ }^{14,15}$, several well designed and convincing studies have clearly shown association between vitamin D deficiency and more severe COVID-19 clinical manifestations ${ }^{16-20,46}$. Recent interventional studies provide further evidence on beneficial and protective impact of vitamin D3 among COVID-19 patients ${ }^{21-24}$. Protective vitamin D3 mechanisms in COVID-19 patients are presumed to be multiple, including potent immunomodulatory activity, antiviral peptide induction, enhancement of physical barrier integrity and even direct interference with viral replication ${ }^{13-15,47}$. Vitamin D-mediated suppression of excessive T helper cell type 1 (Th1) response and proinflammatory cytokine hypersecretion is considered as one of the most important effect that reduces the likelihood of cytokine storm development, major pathogenic event concerning severe COVID-19 manifestation ${ }^{14,15,47-49}$. Promoting induction of the T regulatory cells (Tregs), vitamin D may further enhance these immunomodulatory and anti-inflammatory effects ${ }^{15}$. Ability of vitamin D3 to induce production of type I interferons (IFNs) is also of great importance during SARS$\mathrm{CoV}-2$ infection, since type I IFNs has been known as the most powerful natural mediators of antiviral activity in humans, keeping viral replication under control and enabling effective viral clearance without excessive inflammatory response ${ }^{50}$. In addition, impaired or delayed type I IFNs response during early stage of SARS-CoV-2 infection have been found to play a major role in the cytokine storm development ${ }^{51,52}$, probably by allowing accelerated viral replication, which at a later stage leads to excessive inflammation.

Considering the available data on the protective vitamin D3 effects, but also the "pandemic" of vitamin D deficiency ${ }^{25-28}$, we can assume that in our patient, burdened by multiple risk factors, sufficient plasma 25$\mathrm{OH}$-vitamin D3 curbed detrimental immunopathogenic mechanisms and enabled favorable outcome. Since there are high prevalence of vitamin D3 deficiency in general population, with even more frequent occurrence among patients with obesity, T2DM and hypertension ${ }^{27,28}$, and our patient had not been supplemented per os , we can presume that sufficient level of 25-OH-vitamin D3 had been achieved by UVB phototherapy. This assumption complies to observed seasonal changes in COVID-19 symptom severity and incidence, which are the lesser during the periods with the longer sunlight duration ${ }^{15,53}$. However, such scenario with a complex pathophysiological constellation do not allow us to rule out the possible impact of the methotrexate therapy, as well as the influence of patient's genetic makeup on the course and outcome of the disease. It is also noteworthy that patient gained appropriate antiviral immunoglobulin levels, despite prolonged methotrexate treatment, since it is known that methotrexate can suppress the production of antibodies to neoantigens ${ }^{54-56}$. As vitamin D3 has been found to promote T helper type 2 (Th2) response, IL-4 production and Tregs generation ${ }^{15,57}$, such humoral outcome could also be supported by sufficient $25-\mathrm{OH}$-vitamin D3 status in described patient. Interestingly, an increase in the frequency of naïve B cells and Tregs after narrowband UVB phototherapy was previously shown ${ }^{58}$. These observations may be of great importance for improving COVID-19 vaccination efficacy, but further thorough studies are needed on this topic.

In conclusion, we point to likely possible role of vitamin D as a positive modifier of COVID-19 course and outcome and suggest that routine determination of 25-OH-vitamin D3 status could be considered as useful tool for, at least rough, estimation of COVID-19 outcome, as well as for deciding on vitamin D3 supplementation.

\section{Conflict of Interest}

Both authors declare no conflicts of interest.

\section{Ethical Considerations}

The patient was informed in detail and he provided written consent.

\section{Data Availability Statement}

The data are not publicly available due to the protection of patient privacy and adherence to ethical principles. Clinical and laboratory data may be provided upon reasonable request to corresponding author.

\section{Author Contributions:}


Martina Kralj managed the patient, collected the data, and contributed to the manuscript drafting.

Hrvoje Jakovac interpreted the findings, reviewed the literature, drafted, and wrote the manuscript.

Both authors have read and approved the final manuscript.

\section{References}

1. Yang, X., Yu, Y., Xu J., et al. 2020. Clinical course and outcomes of critically ill patients with SARSCoV-2 pneumonia in Wuhan, China: a single-centered, retrospective, observational study. Lancet Respir Med 8:475-481. doi:10.1016/S2213-2600(20)30079-5.

2. Grasselli, G., Greco, M., Zanella, A., et al. 2020. Risk Factors Associated With Mortality Among Patients With COVID-19 in Intensive Care Units in Lombardy, Italy. JAMA Intern Med 180:13451355. doi:10.1001/jamainternmed.2020.3539.

3. Ahlström, B., Frithiof, R., Hultström, M., et al. 2021. The swedish covid-19 intensive care cohort: risk factors of icu admission and icu mortality. Acta Anaesthesiol Scand 2021;10.1111/aas.13781. doi:10.1111/aas.13781.

4. Rehman, M.F.U., Fariha, C., Anwar, A., et al. 2021. Novel coronavirus disease (COVID-19) pandemic: A recent mini review. Comput Struct Biotechnol J 19:612-623. doi:10.1016/j.csbj.2020.12.033.

5. Apicella, M., Campopiano, M.C., Mantuano, M., et al. 2020. COVID-19 in people with diabetes: understanding the reasons for worse outcomes. Lancet Diabetes Endocrinol 8:782-792. doi:10.1016/S22138587(20)30238-2.

6. Romagnoli, S., Peris, A., De Gaudio, A.R., et al. 2020. SARS-CoV-2 and COVID-19: From the Bench to the Bedside. Physiol Rev 100:1455-1466. doi:10.1152/physrev.00020.2020

7. Mohammad, S., Aziz, R., Al Mahri, S., et al. 2021. Obesity and COVID-19: what makes obese host so vulnerable?. Immun Ageing 18:1. doi:10.1186/s12979-020-00212-x.

8. Abu-Farha, M., Al-Mulla, F., Thanaraj, TA., et al. 2020. Impact of Diabetes in Patients Diagnosed With COVID-19. Front Immunol 11:576818. doi:10.3389/fimmu.2020.576818

9. Sharma, R.K., Li, J., Krishnan, S., et al., 2021. Angiotensin-converting enzyme 2 and COVID-19 in cardiorenal diseases. Clin Sci (Lond) 135:1-17. doi:10.1042/CS20200482.

10. Pasrija, R., Naime, M. 2020. The deregulated immune reaction and cytokines release storm (CRS) in COVID-19 disease. Int Immunopharmacol 90:107225. doi:10.1016/j.intimp.2020.107225.

11. Krishnan, A., Hamilton, .J.P, Alqahtani, S.A., et al. 2021. A narrative review of coronavirus disease 2019 (COVID-19): clinical, epidemiological characteristics, and systemic manifestations. Intern Emerg Med doi: 10.1007/s11739-020-02616-5.

12. Mehta, P., McAuley, D.F., Brown, M., et al. 2020. COVID-19: consider cytokine storm syndromes and immunosuppression. Lancet 395:1033-1034. doi:10.1016/S0140-6736(20)30628-0.

13. Teymoori-Rad, M., Shokri, F., Salimi, V., et al. 2019. The interplay between vitamin D and viral infections. Rev Med Virol 29:e2032. doi:10.1002/rmv.2032.

14. Jakovac H. 2020. COVID-19 and vitamin D-Is there a link and an opportunity for intervention?. Am J Physiol Endocrinol Metab 318:E589. doi:10.1152/ajpendo.00138.2020.

15. Grant, W.B., Lahore, H., McDonnell, S.L., et al. 2020. Evidence that Vitamin D Supplementation Could Reduce Risk of Influenza and COVID-19 Infections and Deaths. Nutrients 12:988. doi: $10.3390 /$ nu12040988.

16. Pereira, M., Dantas Damascena, A., Galvão Azevedo, L.M., et al. 2020. Vitamin D deficiency aggravates COVID-19: systematic review and meta-analysis. Crit Rev Food Sci Nutr 4:1-9. doi:10.1080/10408398.2020.1841090.

17. Jain, A., Chaurasia, R., Sengar, N.S., et al. 2020. Analysis of vitamin D level among asymptomatic and critically ill COVID-19 patients and its correlation with inflammatory markers. Sci Rep 10:20191. doi: 10.1038/s41598-020-77093-z.

18. Mariani, J., Giménez, V.M.M., Bergam, I., et al. 2020. Association Between Vitamin D Deficiency and COVID-19 Incidence, Complications, and Mortality in 46 Countries: An Ecological Study. Health Secur. doi: 10.1089/hs.2020.0137. 
19. Katz, J., Yue, S., Xue, W. 2020. Increased risk for COVID-19 in patients with vitamin D deficiency. Nutrition 84:111106. doi: 10.1016/j.nut.2020.111106.

20. Luo, X., Liao, Q., Shen, Y., et al. 2021. Vitamin D Deficiency Is Inversely Associated with COVID-19 Incidence and Disease Severity in Chinese People. J Nutr 151:98-103. doi:10.1093/jn/nxaa332.

21. Entrenas Castillo, M., Entr.enas Costa, L.M., Vaquero Barrios, J.M, et al. 2020. Effect of calcifediol treatment and best available therapy versus best available therapy on intensive care unit admission and mortality among patients hospitalized for COVID-19: A pilot randomized clinical study. J Steroid Biochem Mol Biol 203:105751. doi:10.1016/j.jsbmb.2020.105751.

22. Ling, S.F., Broad, E., Murphy, R., et al. 2020. High-Dose Cholecalciferol Booster Therapy is Associated with a Reduced Risk of Mortality in Patients with COVID-19: A Cross-Sectional Multi-Centre Observational Study. Nutrients 12:3799. doi: 10.3390/nu12123799.

23. Tan, C.W., Ho, L.P., Kalimuddin, S., et al. 2020. Cohort study to evaluate the effect of vitamin D, magnesium, and vitamin B12 in combination on progression to severe outcomes in older patients with coronavirus (COVID-19). Nutrition 79-80:111017. doi:10.1016/j.nut.2020.111017.

24. Ohaegbulam, K.C., Swalih, M., Patel, P., et al. 2020. Vitamin D Supplementation in COVID-19 Patients: A Clinical Case Series. Am J Ther 27:e485-e490. doi:10.1097/MJT.0000000000001222.

25. Holick, M.F. 2017. The vitamin D deficiency pandemic: Approaches for diagnosis, treatment and prevention. Rev Endocr Metab Disord 18:153-165. doi:10.1007/s11154-017-9424-1.

26. Roth, D.E., Abrams, S.A., Aloia, J., et al. 2018. Global prevalence and disease burden of vitamin D deficiency: a roadmap for action in low- and middle-income countries. Ann N Y Acad Sci 1430:44-79. doi:10.1111/nyas.13968.

27. Liu, X., Baylin, A., Levy, P.D. 2018. Vitamin D deficiency and insufficiency among US adults: prevalence, predictors and clinical implications. Br J Nutr 119.:928-936. doi:10.1017/S0007114518000491.

28. Cashman, K.D., Dowling, K.G., Škrabáková, Z., et al. 2016. Vitamin D deficiency in Europe: pandemic?. Am J Clin Nutr 103:1033-1044. doi:10.3945/ajcn.115.120873.

29. Jakovac H. 2020. COVID-19 and hypertension: is the HSP60 culprit for the severe course and worse outcome?. Am J Physiol Heart Circ Physiol 319:H793-H796. doi:10.1152/ajpheart.00506.2020.

30. Sadeghinia, A., Daneshpazhooh, M. 2020. Immunosuppressive drugs for patients with psoriasis during the COVID-19 pandemic era. A review. Dermatol Ther e14498. doi:10.1111/dth.14498.

31. FAI2R/SFR/SNFMI/SOFREMIP/CRI/IMIDIATE consortium and contributors. 2020. Severity of COVID-19 and survival in patients with rheumatic and inflammatory diseases: data from the French RMD COVID-19 cohort of 694 patients. Ann Rheum Dis doi:10.1136/annrheumdis-2020-218310.

32. Schend, J., Daniels, P., Sanan, N., et al. 2020. Clinical Observation of COVID-19 in a Patient With an Acquired Humoral Deficiency Secondary to Chemotherapeutic Agents. Allergy Rhinol (Providence) 11:2152656720978764. doi:10.1177/2152656720978764.

33. Bernar, B., Kropshofer, G., Crazzolara, R., et al. 2020. SARS-CoV-2 infection in a 7-year-old girl with pancytopenia during acute lymphocytic leukemia maintenance therapy. Pediatr Blood Cancer 67:e28391. doi:10.1002/pbc.28391.

34. Ghazawi, F.M., Lim, M., Dutz, J.P., et al. 2020. Infection risk of dermatologic therapeutics during the COVID-19 pandemic: an evidence-based recalibration. Int J Dermatol 59:1043-1056. doi:10.1111/ijd.15028.

35. Jose, S.S., Bendickova, K., Kepak, T., et al. 2017. Chronic Inflammation in Immune Aging: Role of Pattern Recognition Receptor Crosstalk with the Telomere Complex?. Front Immunol 8:1078. doi:10.3389/fimmu.2017.01078.

36. Bulut, O., Kilic, G., Domínguez-Andrés, J., et al. 2020. Overcoming immune dysfunction in the elderly: trained immunity as a novel approach. Int Immunol 32:741-753. doi:10.1093/intimm/dxaa052.

37. Qin, W.D., Bai, W., Liu, K., et al. 2021. Clinical course and risk factors of disease deterioration in critically ill patients with COVID-19. Hum Gene Ther 10.1089/hum.2020.255. doi:10.1089/hum.2020.255.

38. Lanini, S., Montaldo, C., Nicastri, E., et al. 2020. COVID-19 disease-Temporal analyses of complete blood count parameters over course of illness, and relationship to patient demographics and management outcomes in survivors and non-survivors: A longitudinal descriptive cohort study. PloS one 15: 
e0244129. doi: 10.1371/journal.pone.0244129.

39. Wakkee, M., de Vries, E., van den Haak, P., et al. 2011. Increased risk of infectious disease requiring hospitalization among patients with psoriasis: a population-based cohort. J Am Acad Dermatol 65:1135-1144.

40. Takeshita, J., Shin, D.B., Ogdie, A., et al. 2018. Risk of serious infection, opportunistic infection, and herpes zoster among patients with psoriasis in the United Kingdom. J Invest Dermatol 138.:1726-1735.

41. Hsu, D.Y., Gordon, K., Silverberg, J.I. 2016. Serious infections in hospitalized patients with psoriasis in the United States. J Am Acad Dermatol 75:287-296.

42. Yiu, Z.Z., Parisi, R., Lunt, M., et al. 2020. Risk of hospitalisation and death due to infection in people with psoriasis: a population-based cohort study using the clinical practice research datalink. Br J Dermatol 184:78-86. doi: 10.1111/bjd.19052.

43. Balak, D.M.W., Gerdes, S., Parodi, A., et al. 2020. Long-term Safety of Oral Systemic Therapies for Psoriasis: A Comprehensive Review of the Literature. Dermatol Ther (Heidelb) 10:589-613. doi:10.1007/s13555-020-00409-4.

44. Haustein, U.F., Rytter, M. 2000. Methotrexate in psoriasis: 26 years' experience with low-dose longterm treatment. J Eur Acad Dermatol Venereol 14:382-388. doi:10.1046/j.1468-3083.2000.00058.x.

45. Taneri, P.E., Gomez-Ochoa, S.A., Llanaj, E., et al. 2020. Anemia and iron metabolism in COVID-19: a systematic review and meta-analysis. Eur J Epidemiol 35:763-773. doi:10.1007/s10654-020-00678-5.

46. Maghbooli, Z., Sahraian, M.A., Ebrahimi M., et al. 2020. Vitamin D sufficiency, a serum 25hydroxyvitamin $\mathrm{D}$ at least $30 \mathrm{ng} / \mathrm{mL}$ reduced risk for adverse clinical outcomes in patients with COVID-19 infection. PLoS One 15:e0239799. doi:10.1371/journal.pone.0239799.

47. Aygun, H. 2020. Vitamin D can prevent COVID-19 infection-induced multiple organ damage. Naunyn Schmiedebergs Arch Pharmacol 393:1157-1160. doi: 10.1007/s00210-020-01911-4.

48. Kumar, R., Rathi, H., Haq, A., et al. 2020. Putative roles of vitamin D in modulating immune response and immunopathology associated with COVID-19. Virus Res 292:198235. doi: 10.1016/j.virusres.2020.198235.

49. Ahmed, F. 2020. A Network-Based Analysis Reveals the Mechanism Underlying Vitamin D in Suppressing Cytokine Storm and Virus in SARS-CoV-2 Infection. Front Immunol 11:590459. doi: 10.3389/fimmu.2020.590459.

50. Gauzzi, M.C., Fantuzzi, L. 2020. Reply to Jakovac: COVID-19, vitamin D, and type I interferon. Am J Physiol Endocrinol Metab 319:E245-E246. doi: 10.1152/ajpendo.00315.2020.

51. Pellegrini, S., Uze, G. 2020. An Old Cytokine Against a New Virus? J Interferon Cytokine Res 40:425-428. doi: 10.1089/jir.2020.0130.

52. Kim, J.S., Lee, J.Y., Yang, J.W., et al. 2021. Immunopathogenesis and treatment of cytokine storm in COVID-19. Theranostics 11:316-329. doi: 10.7150/thno.49713.

53. Carleton, T., Cornetet, J., Huybers, P., et al. 2021. Global evidence for ultraviolet radiation decreasing COVID-19 growth rates. Proc Natl Acad Sci U S A 118:e2012370118. doi: 10.1073/pnas.2012370118.

54. Sonani, B., Aslam, F., Goyal, A., et al. 2021. COVID-19 vaccination in immunocompromised patients. Clin Rheumatol 11:1-2. doi: 10.1007/s10067-020-05547-w.

55. Chiricozzi, A., Gisondi, P., Bellinato, F., et al. 2020. Immune Response to Vaccination in Patients with Psoriasis Treated with Systemic Therapies. Vaccines (Basel) 8:769. doi: 10.3390/vaccines8040769.

56. Park, J.K., Lee, Y.J., Bitoun, S., et al. 2019. Interaction between B-cell activation factor and methotrexate impacts immunogenicity of seasonal influenza vaccination in patients with rheumatoid arthritis. Ann Rheum Dis 78:282-284. doi: 10.1136/annrheumdis-2018-214025.

57. Cantorna, M.T., Snyder, L., Lin, Y.D., et al. 2015. Vitamin D and 1,25(OH)2D regulation of T cells. Nutrients 7:3011-3021. doi: 10.3390/nu7043011.

58. Trend, S., Jones, A.P., Cha, L., et al. 2019. Short-term changes in frequencies of circulating leukocytes associated with narrowband UVB phototherapy in people with clinically isolated syndrome. Sci Rep 9:7980. doi: 10.1038/s41598-019-44488-6.

Table 1. Laboratory findings upon detection of SARS-CoV-2 positivity (3 days after symptoms appeared $=$ median time of symptoms duration) 


\begin{tabular}{|c|c|c|}
\hline Parameter (unit) & Result & Reference range \\
\hline $\begin{array}{l}\text { Total white blood cell }\left(\times 10^{9} / \mathrm{L}\right) \\
\end{array}$ & 4.8 & $3.4-9.7$ \\
\hline Lymphocytes $\left(\right.$ x $\left.10^{9} / \mathrm{L}\right)$ & 1.02 & $1.19-3.35$ \\
\hline Neutrophils $\left(\right.$ x $\left.10^{9} / \mathrm{L}\right)$ & 3.10 & $2.06-6.49$ \\
\hline Monocytes (x $\left.10^{9} / \mathrm{L}\right)$ & 0.50 & $0.12-0.84$ \\
\hline Eosinophils $\left(\mathrm{x} 10^{9} / \mathrm{L}\right)$ & 0.15 & $0.00-0.43$ \\
\hline Basophils $\left(\right.$ × $\left.10^{9} / \mathrm{L}\right)$ & 0.04 & $0.00-0.06$ \\
\hline Red blood cell (x 10 $12 / \mathrm{L})$ & 4.06 & $4.34-5.72$ \\
\hline Hematocrit (\%) & 0.387 & $0.415-0.530$ \\
\hline Hemoglobin (g/L) & 132 & $138-175$ \\
\hline $\mathrm{MCV}(\mathrm{fL})$ & 95.3 & $83.0-97.2$ \\
\hline $\mathrm{MCH}(\mathrm{pg})$ & 32.5 & $27.4-33.9$ \\
\hline $\operatorname{MCHC}(\mathrm{g} / \mathrm{L})$ & 341 & $320-345$ \\
\hline Platelets $\left(\mathrm{x} 10^{9} / \mathrm{L}\right)$ & 240 & $158-424$ \\
\hline MPV (fL) & 9.7 & $6.8-10.4$ \\
\hline Prothrombin time & 0.96 & $>0.7$ \\
\hline C-reactive protein $(\mathrm{mg} / \mathrm{L})$ & 7 & $0-5$ \\
\hline Erythrocyte sedimentation rate $-1 \mathrm{~h}(\mathrm{~mm} / 3.6 \mathrm{ks})$ & 50 & $3-23$ \\
\hline Glucose $(\mathrm{mmol} / \mathrm{L})$ & 10.1 & $4.4-6.4$ \\
\hline Triglycerides (mmol/L) & 1.8 & $<1.7$ \\
\hline Total cholesterol (mmol/L) & 3.6 & $<5.0$ \\
\hline LDL cholesterol (mmol/L) & 2.1 & $<3.0$ \\
\hline HDL cholesterol (mmol/L) & 1.1 & $>1.0$ \\
\hline Urea (carbamide) (mmol/L) & 7.5 & $2.8-8.3$ \\
\hline Creatinine $(\mu \mathrm{mol} / \mathrm{L})$ & 134 & $64-104$ \\
\hline Total bilirubin $(\mu \mathrm{mol} / \mathrm{L})$ & 10 & $3-20$ \\
\hline 25-OH-vitamin D3 (nmol/L) & 92.2 & $50.0-125.0$ \\
\hline Urinary proteins (dipstick) & $1+$ & negative \\
\hline Urinary glucose (dipstick) & negative & negative \\
\hline $\mathrm{SpO} 2$ by pulse oximeter $(\%)$ & 95 & $>94$ \\
\hline
\end{tabular}

